\title{
Tunisian population quality of life: a general analysis using SF-36
}

Safa Salem, ${ }^{1}$ Dhafer Malouche ${ }^{2}$ and Habiba Ben Romdhane ${ }^{3}$

${ }^{1}$ Ecole Supérieure de la Statistique et de l'Analyse de l'Information, Tunisia. ${ }^{2}$ Dhafer Malouche, Unité Modelisation, Analyse Statistique et Economique, Tunisia. ${ }^{3}$ Laboratoire d'Epidémiologie, Faculté de Medecine de Tunis, Tunisia. (Correspondence to: Safa Salem: safa.salemo4@gmail.com).

\begin{abstract}
Background: The SF-36 is the instrument for measuring the health relatedquality of life (HRQOL) of patients in many clinical and national studies to describe the health status of populations, by obtaining comparable data on health status internationally.

Aims: This study aimed to obtain population norms for the Tunisian version of SF-36 and to assess the association between socio HRQOL scores with the demographic characteristics of the Tunisian population.

Methods: Face-to-face interviews for a cross-sectional study were carried out in 2005 to collect socio demographic and environmental variables as well as self-reported quality of life. A representative sample of 6543 aged between 35 and 70 years old were selected.

Results: All scores had a high level of internal consistency reliability coefficient. HRQOL score levels were associated with sociodemographic characteristics and a decrease as age increased. The averages of the physical and mental component summary were $53+/-8$ for males and $47 \cdot 7+/-10$ for females.

Conclusions: This study was the first to address the general Tunisian population. This study shed light on factors associated with HRQOL in the Tunisian context.

Keywords: Quality of life, SF-36, Tunisia, HRQOL, population

Citation: Salem S; Malouche D; Romdhane H. Tunisian population quality of life: a general analysis using SF-36. East Mediterr Health J.2019;25(9):613621 https://doi.org/10.26719/emhj.18.030

Received: 21/11/16; accepted: 01/10/17

Copyright @ World Health Organization (WHO) 2019. Some rights reserved. This work is available under the CC BY-NC-SA 3.0 IGO license (https:// creativecommons.org/licenses/by-nc-sa/3.0/igo)
\end{abstract}

\section{Introduction}

Since 1948, the World Health Organization (WHO) has defined health as a "state of complete physical, mental and social well-being and not merely the absence of disease or infirmity". Beyond the indication of change in the frequency and severity of disease, the measurement of health and effect of health care must include an estimation of well-being and this can be assessed by measuring the improvement in the quality of life related to health care (1). In 1995, WHO recognized the importance of evaluating and improving people's quality of life.

WHO defined health-related quality of life (HRQOL) as individuals' perceptions of their position in life in the context of culture and value systems in which they live and in relation to their goals, expectations, standards and concerns (1). Since health is a multidimensional concept, HRQOL is also multidimensional with domains related to physical, mental and emotional, and social functioning.

In 1995, WHO recognized the importance of evaluating and improving people's quality of life. The HRQOL may be measured using several instruments such as SF-36, which was used in this study. The Medical Outcomes Study 36-Item Short Form (SF-36) is a generic measure of health status, providing scores on eight areas of functioning and well-being as well as two broad areas of subjective well-being, namely physical health and mental health. It can be used in diseased groups as well as in general populations and it has been considered as a consistent and good measure of differences between groups defined by age, sex, socio-economic status, geographical region and clinical conditions. In response to criticism that the SF-20 was too brief to capture changes in health status, the SF-36 was designed $(2,3)$. To obtain comparable data on health status internationally, SF-36 has been translated into various languages and adapted to different cultures (4).

Similarly, the residence area or regions may affect the quality of life of the population, such as in Lebanon, where there is a significant difference between urban and rural residential areas. Moreover, people in better social positions are expected to have a better quality of life. These hypotheses have been revealed in a number of studies from high-income countries (5) and in lowand middle-income countries (6).

The SF-36 questionnaire was the instrument for measuring the quality of life of patients in many national studies, for both healthy and unhealthy people (5-8). In Tunisia several studies covered subgroups of unhealthy people who were suffering from specific diseases (9-11). This study is the first to address the general population in Tunisia. It aims to obtain population norms for the Tunisian version of SF-36 and to assess the variation of self-reported quality of life according to different demographic and socioeconomic characteristics. A translated and validated version of SF-36 was used. 


\section{Methods}

\section{Study area}

Tunisia is a North African country situated between Algeria and Libya. At $163000 \mathrm{~km}^{2}$, it is the smallest country in North Africa with a population of 10 million (of which about two thirds are urban). Life expectancy at birth is 74 years for men and 78 for women. The Tunisian gross domestic product per capita (expressed as purchase parity power, [PPP]) reached US\$ 10 439.70, the highest in North Africa. The Human Development Index was estimated at 0.721 in 2014, ranking the country at 90, placing it ahead of Algeria (94) and Morocco (129) (12). SF-36 was administered in a cross-sectional study to collect sociodemographic and environmental variables as well as self-reported quality of life. A representative sample was analysed.

\section{Target population and sampling}

The national cross-sectional survey was carried out from April to September 2005. The target population was all Tunisian adults aged 35 to 70 years. It was based on a national stratified three stage cluster sample of subjects; the sampling frame was derived by the Tunisian Institute of Statistics from the database of the most recent census of the population carried out in 2004. Stratification was according to the seven administrative regions that divide Tunisia, and each region being a stratum. The first and second stage of random selection were performed using the national census database: in each of the seven strata, 47 census districts were randomly selected at the first stage, with a probability proportional to size in number of eligible households (i.e., featuring at least one 35-70 year old subject). At the second stage, 25 eligible households were randomly sampled in each district. The third stage of selection was performed during the implementation of the field survey: in each household one subject from the targeted age was included in the survey.

The study protocol was carried out according to the Declaration of Helsinki and was ethically approved by the Tunisian Ministry of Health and the Tunisian National Council of Statistics. All participants gave their free informed consent after being thoroughly informed of the purpose, requirements and procedures of the survey. A total of 8184 people were enrolled. Of these, 1651 individuals had missed answering the total SF-36 questionnaire or at least half. The remaining 6543 participants (2832 men and 3557 women) constituted the sample used. The mean age of the 6543 individuals (49.14 years for men, 50.26 years for women) was similar to that of the initial 8184 individuals recruited for the survey (49.6 years for men, 49.5 years for women).

\section{Measures}

Health-related quality of life was measured by a translated and validated version of the SF-36, which contains 36 items covering eight dimensions of perceived health: general health perceptions (5 items), physical functioning (10 items), role limitations because of physical func- tioning (4 items), bodily pain ( 2 items), general mental health ( 5 items), role limitations because of emotional problems ( 3 items), vitality ( 4 items), and social functioning ( 2 items). The remaining item, relating to change in health, is not scored as a separate dimension. For each dimension of the SF-36, the items were coded, aggregated, and transformed on a scale ranging from o (the worst health status) to 100 (the best health status), as described in the scoring manual (13). Scores were calculated whenever there was information for at least half of the items of the dimension.

The SF-36 summary is based on two components summary: mental component summary (MCS) and physical component summary (PCS), and is measured in three-steps: 1) the eight computed sub-scale scores (range 0-100) are standardized using a linear Z-score transformation. Z-scores are calculated by subtracting subscale means for the Tunisian population sample from each individual's sub-scale scores and dividing the difference by the standard deviation of the Tunisian sample. 2) Z-scores are multiplied by the sub-scale factor score coefficients (Tables 1-8) for PCS and MCS and summed over all eight sub-scales. These coefficients were obtained with a PCA and a varimax rotation. 3) t-scores are calculated by centering the obtained PCS and MCS sums to get a mean of 50 and a standard deviation equal to 10 (14).

\begin{tabular}{|c|c|}
\hline \multicolumn{2}{|c|}{ Table 1 Sample description } \\
\hline Covariates & $\mathbf{N}(\%)$ \\
\hline \multicolumn{2}{|l|}{ Gender } \\
\hline Male & $2832(44.32)$ \\
\hline Female & $3557(55.67)$ \\
\hline \multicolumn{2}{|l|}{ Age } \\
\hline $35-44$ & $2527(39.55)$ \\
\hline $45-54$ & $2029(31.75)$ \\
\hline $55-64$ & $1121(17.54)$ \\
\hline $65-74$ & $712(10.88)$ \\
\hline \multicolumn{2}{|l|}{ Educational level } \\
\hline Illiterate & $2762(43.23)$ \\
\hline Primary school & $2150(33.65)$ \\
\hline Secondary school & $1041(16.29)$ \\
\hline Short education & $100(1.56)$ \\
\hline University & $313(4.89)$ \\
\hline \multicolumn{2}{|l|}{ Milieu } \\
\hline Urban & $3669(57.42)$ \\
\hline Rural & $2720(42.57)$ \\
\hline \multicolumn{2}{|l|}{ Activity } \\
\hline Active & $2615(40.92)$ \\
\hline Inactive & $3650(57.12)$ \\
\hline \multicolumn{2}{|l|}{ Economical level } \\
\hline Low & $2369(37.07)$ \\
\hline Median & $2147(33.60)$ \\
\hline High & $1539(24.08)$ \\
\hline
\end{tabular}




\section{Statistics analyses}

All statistical analyses were conducted using R statistical software package 3.0.1. The mean and standard deviation (SD) for responses to each scale were calculated. A multiple comparison of treatments by means of Tukey was performed to test the effect of age on all domains of the SF-36. The level by alpha default is 0.05 . As a first step in our analyses we described the sample by its demographic characteristics, which are age, sex, educational level, residence area and activity. The second step was to calculate the eight sub-scales and the two component summary using Tunisian coefficients and to describe the sample by their means +/- SD of the eight sub-scales and the two component summary.

A linear Bayesian regression was used to assess relation between scales and socio-demographic characteristics of individuals. Bayesian regression is more flexible when we want to use certain prior information on the process of collecting the data, in contrast to frequentist regression where there is no way to use this prior information. Since there was no reliable prior information, non-informative was used prior when performing the Bayesian regression.

Results are reported as posterior mean differences with $95 \%$ credible intervals (Bayesian analogue to frequentist confidence intervals). The SF-36 domain score for each subject is assumed to follow a normal distribution with mean $\mu$ i and variance $\tau$ that is:

$$
\mathrm{Yi} \sim \mathrm{N}(\mu \mathrm{i}, \tau) ; \mu \mathrm{i}=\sum_{j=1}^{p} \beta \mathrm{j} \mathrm{Xij}
$$

\section{i: 1..n, n: individual number}

j: 1..p, p: variable number

Yi: The health score (PCS and MCS)

Xij: Independent variables ( Age, sex, educational level, economical level)

$B j$ : a set of coefficients, one each for each X.

Only SF-36 questionnaires with more than $50 \%$ of responses were considered as valid and investigated in our study. R software glmulti Package, which is an automated model selection and model averaging software that automatically generates all possible outcomes within the specified response and explanatory variables, and producing best models in terms of Information Criterion (AIC, AICc or BIC), was used to determine the best model with interaction between social determinants using the genetic algorithm and the AIC criteria. (15)

\section{Results}

The mean age of the study population was $49.77 \pm 9.83$. Eleven percent of the study group was over 65 years old. Women were significantly older than men. Almost half of the population was illiterate and only $5 \%$ of the study population achieved higher education. $57 \%$ of the study pop-
Table 2 Descriptive statistics for SF-36 scales in the general Tunisian population

\begin{tabular}{cccc} 
Scales & Mean & $\begin{array}{c}\text { Standard } \\
\text { deviation }\end{array}$ & $\begin{array}{c}\text { Cronbach } \\
\text { Alpha }\end{array}$ \\
PF & 82.13 & 23.63 & 0.923 \\
RP & 70.65 & 40.67 & 0.917 \\
BP & 67.98 & 33.44 & 0.922 \\
GH & 56.43 & 20.91 & 0.804 \\
VT & 57.95 & 22.66 & 0.796 \\
SF & 78.74 & 27.86 & 0.805 \\
RE & 75.46 & 40.13 & 0.925 \\
MH & 65.56 & 21.49 & 0.829 \\
PCS & 50.09 & 9.93 & - \\
MCS & 50.07 & 9.95 & - \\
\hline
\end{tabular}

PF = Physical functioning; $R P=$ Role physical $; \mathrm{BP}=$ Bodily pain; $\mathrm{GH}=$ General health; $\mathrm{VT}=$ Vitality; SF = Social functioning; RE = Role emotional; $M H=$ Mental health; $P C S=$ Physical Component Summary; MCS = Mental Component Summary

\begin{tabular}{cccc}
\hline Table 3 Gender based comparison for each scale & \\
Scales & Male & Female & Pvalue \\
PF & $88.82(18.77)$ & $77.16(25.39)$ & $<0.001$ \\
RP & $79.76(35.93)$ & $63.89(42.54)$ & $<0.001$ \\
BP & $75.11(30.96)$ & $62.72(34.25)$ & $<0.001$ \\
GH & $61.68(19.82)$ & $52.46(20.76)$ & $<0.001$ \\
VT & $64.31(21.79)$ & $53.19(21.98)$ & $<0.001$ \\
SF & $85.42(23.87)$ & $73.87(29.43)$ & $<0.001$ \\
RE & $82.16(42.39)$ & $70.50(42.39)$ & $<0.001$ \\
MH & $69.96(20.24)$ & $62.16(21.75)$ & $<0.001$ \\
PCS & $53.06(8.55)$ & $47.72(10.31)$ & $<0.001$ \\
MCS & $52.96(8.99)$ & $47.78(10.08)$ & $<0.001$ \\
\hline
\end{tabular}

ulation lived in urban areas and almost $60 \%$ were non-active. Participants that rated a high economical level were $24 \%$ while $37 \%$ rated a low economical level (Table 1). Table 2 presents descriptive statistics for SF-36 scales in the general Tunisian population, and includes the mean, the standard deviation and the Cronbach alpha test.

All scales presented in Table 2 have a high level of internal consistency reliability coefficient. PCS values ranged from 18 to 64.03 and MCS values ranged from 17.06 to 67.88 with median values 53.36 and 52.21 for PCS and MCS respectively. Figure 1 represents the mean value of eight scales. The physical functioning scale is the highest while vitality scale and general health were the lowest. Men had significantly higher scores than women for the eight domains (Table 3). Also for the components summary, men had significantly better physical and mental component summary than women (Figure 2). Scores decreased while the age increased. The difference of scores in each age group is significant for the eight domains except the mental health scale we found that there is no significant difference between the 55-64 and 65-74 age groups (Table 4). 


\begin{tabular}{|c|c|c|c|c|}
\hline Scales & $35-44$ yrs & $45-54$ yrs & $55-64$ yrs & $65-74$ yrs \\
\hline $\mathrm{PF}$ & $90.70(16.08) \mathrm{a}$ & $84.18(20.82) b$ & $73.50(26.40) \mathrm{c}$ & $61.36(29.13) d$ \\
\hline $\mathrm{RP}$ & $81.53(44.87) a$ & $72.80(39.42) b$ & $60.07(43.65) \mathrm{c}$ & $44.87(44.88) d$ \\
\hline BP & $75.08(30.82) a$ & $68.14(32.63) b$ & $60.26(34.79) \mathrm{c}$ & $56.01(35.96) d$ \\
\hline $\mathrm{GH}$ & $62.56(19.04) \mathrm{a}$ & $56.37(20.08) b$ & $50.31(21.40) \mathrm{c}$ & $45.51(20.89) d$ \\
\hline VT & $63.58(20.39) a$ & $58.94(21.74) b$ & $51.89(23.10) c$ & $46.16(24.55) d$ \\
\hline SF & $85.52(22.56) a$ & 79.49 (27.00) b & $73.52(29.91) \mathrm{C}$ & $62.93(33.80) d$ \\
\hline RE & $81.57(35.94) a$ & $81.57(38.85) b$ & $69.19(42.53) \mathrm{c}$ & $59.75(46.61) d$ \\
\hline $\mathrm{MH}$ & $68.17(20.44) a$ & $65.26(21.55) \mathrm{b}$ & $63.16(22.05) \mathrm{c}$ & $61.49(22.55) \mathrm{c}$ \\
\hline PCS & $53.37(7.76) \mathrm{a}$ & $50.52(9.28) b$ & $46.68(10.75) c$ & $42.53(11.39) \mathrm{d}$ \\
\hline MCS & $52.72(8.56) \mathrm{a}$ & $50.26(9.64) \mathrm{b}$ & $47.31(10.48) c$ & $44.47(11.08) \mathrm{d}$ \\
\hline
\end{tabular}

The difference between scores is statistically significant if the letters indicated in brackets ( $a, b, c$ and $d)$ are different (Tukey test).

\begin{tabular}{|c|c|c|c|c|}
\hline \multicolumn{5}{|c|}{$\begin{array}{l}\text { Table } 5 \text { Factor loads obtained from Tunisian and US } \\
\text { algorithms }\end{array}$} \\
\hline Scales & $\begin{array}{l}\text { Factor } 1 \\
\text { (TN) }\end{array}$ & $\begin{array}{l}\text { Factor } 1 \\
\text { (US) }\end{array}$ & $\begin{array}{c}\text { Factor } 2 \\
\text { (TN) }\end{array}$ & $\begin{array}{c}\text { Factor } 2 \\
\text { (US) }\end{array}$ \\
\hline $\mathrm{PF}$ & 0.85 & 0.88 & 0.19 & 0.04 \\
\hline $\mathrm{RP}$ & 0.65 & 0.78 & 0.12 & 0.30 \\
\hline BP & 0.29 & 0.77 & 0.25 & 0.24 \\
\hline $\mathrm{GH}$ & 0.60 & 0.68 & 0.57 & 0.32 \\
\hline VT & 0.48 & 0.56 & 0.70 & 0.57 \\
\hline SF & 0.48 & 0.44 & 0.42 & 0.71 \\
\hline $\mathrm{RE}$ & 0.23 & 0.19 & 0.26 & 0.81 \\
\hline $\mathrm{MH}$ & 0.07 & 0.12 & 0.89 & 0.90 \\
\hline
\end{tabular}

The result of varimax rotation solution for Tunisian SF-36 and US loads were presented in Table 5. Factor 1 was used to calculate PCS and factor 2 to calculate MCS. Subjects with higher educational level have higher PCS and MCS (Figure 3 and Figure 4). Bayesian regression shows that all models converged to admissible solutions and all socio-demographics characteristics are significantly associated with health scores (Table 7).

In the final models, the scores vary significantly between sex and age for all dimensions. However, the variation within education levels and economic situation levels were not significant for all dimensions. There was an interaction between education level and sex for the majority of mental health dimensions and for the physical health dimension (RP). In addition, there was an interaction between education level and age for the majority of physical health dimensions. Interaction between economic levels and education and sex exist only for BP dimension but interaction between economic level and age exists and is significant for the majority of dimensions (Table 8).

\section{Discussion}

This is the largest study in Tunisia that aimed to assess the HRQOL of the general Tunisian population and to establish norms for regional and international comparisons. This study provided data on the HRQOL, according to geographical, social, and economic characteristics among adult Tunisian population.

Our results show variability in the different dimensions of the SF-36 for the general population. The highest score for the Tunisian population was the physical functioning and the lowest one was the general health dimension. This result was consistent with other studies conducted in a number of high-income countries such as the United States of America (13), Switzerland (7) and the United Kingdom (2). However, it was different from other studies; for instance, in an Australian study it was found that the highest score was social functioning (16) and for the majority of previous studies in highincome and low- and middle-income countries the lowest scale was vitality $(4,7,13,16)$.

It was observed that four out of eight of SF-36 dimensions have a Cronbach alpha coefficient up to 0.9, which is higher than that found in a Norwegian and Turkish study (the Cronbach Alpha was up to 0.9 only for two out of eight) $(3,4)$. The Cronbach alpha coefficients that are equal to 0.9 and 0.7 are the generally accepted values for individuals and group comparisons (17).

Statistical tests confirm the significant difference of quality of life according to individuals' characteristics such as sex, educational levels and economic situation.

The present study revealed that women report significantly worse scores than men in all SF-36 scales and components summary, which is consistent with findings in other studies in high-income countries $(13,16,18)$, and in low- and middle-income countries (4). Women's lower HQOL might be related to socio-economic situations, sexual taboos and traditional roles (6). In this study, women and illiterates have the worst HRQOL; however, there was no significant difference between secondary school level and university. These results are similar to a Spanish study showing that the level of health was higher among those with high levels of education.

Similar to other studies that have shown that health was worse in older age groups $(4,7,13,16,18)$, these 


\begin{tabular}{cccccc}
\hline \multicolumn{2}{c}{ Table 6 Variation of scores by scales by educational level } & & & \\
Scales & Illiterate & Primary school & Secondary school & Short education & University \\
\hline PF & $74.11 \mathrm{c}$ & $87.08 \mathrm{~b}$ & $90.18 \mathrm{ab}$ & $91.44 \mathrm{ab}$ & $93.04 \mathrm{a}$ \\
RP & $59.95 \mathrm{c}$ & $76.26 \mathrm{~b}$ & $82.47 \mathrm{a}$ & $89 \mathrm{a}$ & $87.17 \mathrm{a}$ \\
BP & $60.38 \mathrm{~b}$ & $72.69 \mathrm{a}$ & $74.91 \mathrm{a}$ & $80.65 \mathrm{a}$ & $80.23 \mathrm{a}$ \\
GH & $49.82 \mathrm{c}$ & $60.56 \mathrm{~b}$ & $62.37 \mathrm{ab}$ & $67.07 \mathrm{a}$ & $65.32 \mathrm{a}$ \\
VT & $50.67 \mathrm{c}$ & $62.11 \mathrm{~b}$ & $65.83 \mathrm{a}$ & $69.9 \mathrm{a}$ & $67.06 \mathrm{a}$ \\
SF & $71.43 \mathrm{~b}$ & $83.71 \mathrm{a}$ & $85.97 \mathrm{a}$ & $87.1 \mathrm{a}$ & $87.36 \mathrm{a}$ \\
RE & $68.96 \mathrm{~b}$ & $79.6 \mathrm{a}$ & $81.98 \mathrm{a}$ & $80.66 \mathrm{a}$ & $85.41 \mathrm{a}$ \\
MH & $60.76 \mathrm{c}$ & $67.25 \mathrm{~b}$ & $71.64 \mathrm{a}$ & $77.08 \mathrm{a}$ & $73.42 \mathrm{a}$ \\
PCS & $46.82 \mathrm{c}$ & $51.98 \mathrm{~b}$ & $53.01 \mathrm{a}$ & $54.53 \mathrm{a}$ & $54.46 \mathrm{a}$ \\
MCS & $46.66 \mathrm{c}$ & $51.68 \mathrm{~b}$ & $53.50 \mathrm{a}$ & $55.57 \mathrm{a}$ & $54.62 \mathrm{a}$ \\
\hline
\end{tabular}

The difference between scores is statistically significant if the letters indicated in brackets ( $a, b, c$ and $d$ ) are different (Tukey test).

results demonstrated that HRQOL increased while age decreased, which may possibly be related to cumulative health problems.

A Bayesian multiple regression analysis was used to ascertain the relation between all sociodemographic characteristics and the dimensions of SF36 and components summary. This multivariate analysis confirmed that sociodemographic characteristics and economic of individuals are independent factors for determining HRQOL scores. All educational levels have significantly higher scores than illiterate persons. HRQOL scores decreases while age is increasing. PCS is decreasing 0.3 with each age year increase, while MCS decreases 0.2 with each age year increase. This indicates that physical health is more affected by age than mental health.

Sex is also an important factor for determining HRQOL scores. Women have lowest HRQOL mental and physical scores, but this sex-based difference is more important for physical health and may be due to the traditional roles of women of raising children and taking care of families. An inverse relation between socioeconomic level and health has been found in this study. People in lower economical level have significantly worst scores than those in higher socioeconomic levels. This has been shown in several studies and is one of the most consistent observations in the history of public health research, indicating that those in lower socioeconomic strata have higher mortality and more frequent health problems than those in higher socioeconomic strata (5).

A genetic algorithm with AIC criteria was used to find out the best models with interaction between social indicators. In our final models variation of scores between education levels was not significant for $\mathrm{GH}$ and VT dimensions, but this variation increase significantly with age. For the other dimensions difference between education levels was significant, and higher for the majority of males in the mental dimension and for the RP dimension. The difference in scores between education levels increases with age increase for the majority of those in the physical dimension and for the SF dimension. The difference of scores between primary school and illiterate, and secondary school and illiterate,
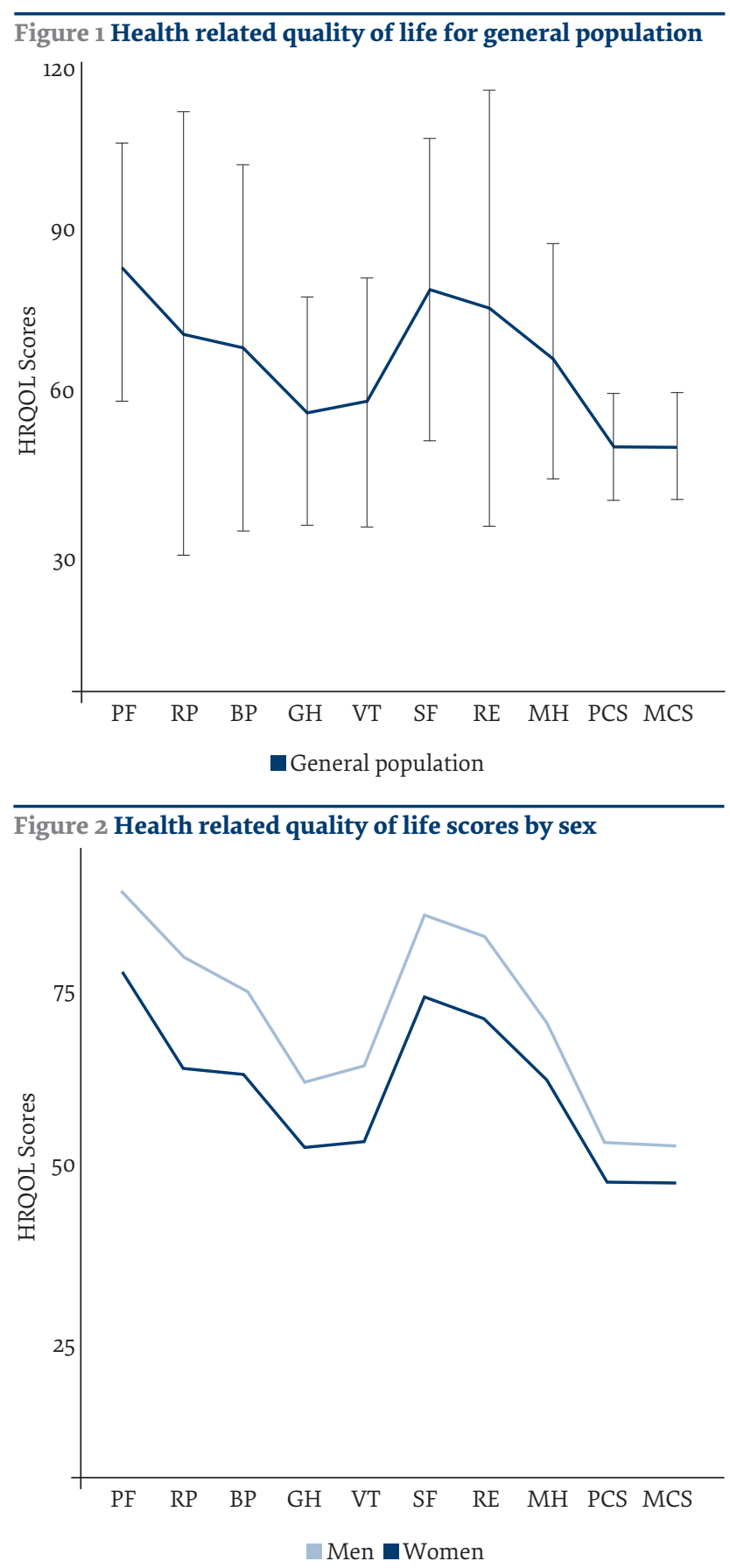


\begin{tabular}{|c|c|c|c|c|c|c|c|c|c|c|}
\hline Factors & PF & $\mathbf{R P}$ & BP & GH & VT & SF & $\mathbf{R E}$ & MH & PCS & MCS \\
\hline Primary school & $2.8^{*}$ & $3^{*}$ & $2.9^{*}$ & $3.3^{*}$ & $3.3^{*}$ & $3.3^{*}$ & $1.8^{*}$ & $1.8^{*}$ & $1.4^{*}$ & $1.4^{*}$ \\
\hline Secondary school & $3.9^{*}$ & $6.2^{*}$ & $1.2^{*}$ & $2.9^{*}$ & $2.9^{*}$ & $2.8^{*}$ & $2.0^{*}$ & $3.1^{*}$ & $1.6^{*}$ & $1.6^{*}$ \\
\hline Short education & $4.8^{*}$ & $10.3^{*}$ & $5 \cdot 4^{*}$ & $7 \cdot 3^{*}$ & $7 \cdot 3^{*}$ & $3.4^{*}$ & $1.4^{*}$ & $8.3^{*}$ & $2.9^{*}$ & $3.4^{*}$ \\
\hline University & $5 \cdot 4^{*}$ & $8.3^{*}$ & $3.8^{*}$ & $4.2^{*}$ & $4.2^{*}$ & $2.6^{*}$ & $4.3^{*}$ & $3.5^{*}$ & $2.1^{*}$ & $1.9^{*}$ \\
\hline Age & $-0.9^{*}$ & $-1.1^{*}$ & $-0.6^{*}$ & $-0.5^{*}$ & $-0.5^{*}$ & $-0.6^{*}$ & $-0.6^{*}$ & -0.1 * & $-0.3^{*}$ & $-0.2^{*}$ \\
\hline Women & $-9.2^{*}$ & $-12.4^{*}$ & $-10.4^{*}$ & $-7.2^{*}$ & $-7.2^{*}$ & $-9.7^{*}$ & $-9.8^{*}$ & $-6.1^{*}$ & $-4.3^{*}$ & $-4.1^{*}$ \\
\hline Median economic level & $1.3^{*}$ & $0.4^{*}$ & $2.2^{*}$ & $3.7^{*}$ & $3.7^{*}$ & $3.3^{*}$ & $1.2^{*}$ & $6.0^{*}$ & $1.2^{*}$ & $2.1^{*}$ \\
\hline High economic level & $1.2^{*}$ & $3.4^{*}$ & $6.9^{*}$ & $4.7^{*}$ & $4.7^{*}$ & $4.7^{*}$ & $2.4^{*}$ & $8.2^{*}$ & $1.8^{*}$ & $3.0^{*}$ \\
\hline Sigma2 & $423.7^{*}$ & $1429.5^{*}$ & $1021.8^{*}$ & $373.9^{*}$ & $373.9^{*}$ & $685.3^{*}$ & $1525.2^{*}$ & $423.1^{*}$ & $78.0^{*}$ & $83.1^{*}$ \\
\hline
\end{tabular}

Sigma 2 is the variance of error term of the regression.

Table 8 Linear regression of 8 scales, physical and mental component summary by social demographic determinants

\begin{tabular}{|c|c|c|c|c|c|c|c|c|c|c|}
\hline Factors & PF & $\mathbf{R P}$ & BP & GH & VT & SF & $\mathbf{R E}$ & MH & PCS & MCS \\
\hline Primary school & $-11^{*}$ & $-20.6^{*}$ & -0.99 & & & 1.18 & $-13.15^{*}$ & $-0.05^{*}$ & $-3.42^{*}$ & 0.56 \\
\hline Secondary school & $-20.94^{*}$ & $-27.98^{*}$ & -4.92 & & & 0.59 & $-37.4^{*}$ & 1.06 & $-6.38^{*}$ & 0.65 \\
\hline Short education & $-29.33^{*}$ & -36.45 & 14.7 & & & 6.79 & $-48.91^{*}$ & $10.72^{*}$ & -9.8 & $4.14^{*}$ \\
\hline University & $-14.61^{*}$ & -21.88 & 16.17 & & & 2.98 & $-39.26^{*}$ & 3.37 & $-699^{*}$ & 1.77 \\
\hline Male & $-10.50^{*}$ & $-15.98^{*}$ & $-8.01^{*}$ & $-4.88^{*}$ & -4.64 & $-24.4^{*}$ & $14.06^{*}$ & $-13.94^{*}$ & $-3.14^{*}$ & -7.21 \\
\hline Medium economic level & $1.68^{*}$ & & -14.03 & $2.64^{*}$ & -0.81 & -4.94 & & & & 0.35 \\
\hline High economic level & $1.5^{*}$ & & $-24.65^{*}$ & $4.67^{*}$ & $-13.56^{*}$ & $-11.57^{*}$ & & & & -3.27 \\
\hline Age & $-1.20^{*}$ & $-1.56^{*}$ & $-0.97^{*}$ & $-0.60^{*}$ & $-0.73^{*}$ & $-1.01^{*}$ & $-1.01^{*}$ & $-0.36^{*}$ & $-0.45^{*}$ & -0.36 \\
\hline Primary school: male & & $6.84^{*}$ & & & & $5 \cdot 46^{*}$ & $7.69^{*}$ & $5.27^{*}$ & & $2.18^{*}$ \\
\hline Secondary school: male & & 2.3 & & & & $5.97^{*}$ & 4.06 & $5.27^{*}$ & & $2.47^{*}$ \\
\hline Short education: male & & -4.67 & & & & -0.83 & 4.56 & -0.02 & & 0.38 \\
\hline University: male & & -0.47 & & & & 2.76 & -3.69 & 2.91 & & 1.45 \\
\hline Primary school: age & $0.26^{*}$ & $0.40^{*}$ & & & & & & 0.21 & & $0.09^{*}$ \\
\hline Secondary school: age & $0.51^{*}$ & $0.70^{*}$ & & & & & & $0.79^{*}$ & & $0.16^{*}$ \\
\hline Short education: age & $0.73^{*}$ & $1.1^{*}$ & & & & & & $1.04^{*}$ & & $0.27^{*}$ \\
\hline University: age & $0.41^{*}$ & $0.66^{*}$ & & & & & & $1.01^{*}$ & & $0.19^{*}$ \\
\hline Primary school: medium economic level & & & $6.73^{*}$ & & & & & & & \\
\hline Secondary school: medium economic level & & & $7.28^{*}$ & & & & & & & \\
\hline Short education: medium economic level & & & -12.3 & & & & & & & \\
\hline University: medium economic level & & & -3.82 & & & & & & & \\
\hline Primary school: high economic level & & & $9.18^{*}$ & & & & & & & \\
\hline Secondary school: high economic level & & & $13.33^{*}$ & & & & & & & \\
\hline Short education: high economic level & & & -2.28 & & & & & & & \\
\hline University: high economic level & & & -7.01 & & & & & & & \\
\hline Male: medium economic level & & & & $2.85^{*}$ & $2.16^{*}$ & & & & & \\
\hline Male: high economic level & & & & 0.36 & $4.03^{*}$ & & & & & \\
\hline Age: primary school & & & & $0.06^{*}$ & $0.07^{*}$ & & & & & \\
\hline Age: secondary school & & & & $0.06^{*}$ & $0.09^{*}$ & & & & & \\
\hline Age: short education level & & & & $0.16^{*}$ & $0.16^{*}$ & & & & & \\
\hline Age: university & & & & $0.09^{*}$ & $0.08^{*}$ & & & & & \\
\hline Age: male & $0.39^{*}$ & $0.51^{*}$ & $0.37^{*}$ & $0.22^{*}$ & $0.23^{*}$ & $0.62^{*}$ & $0.40^{*}$ & $0.33^{*}$ & $0.15^{*}$ & $0.20^{*}$ \\
\hline Age: medium economic level & & 0.02 & & & & & 0.03 & $0.12^{*}$ & $0.02^{*}$ & \\
\hline Age: high economic level & & $0.07^{*}$ & & & & & $0.06^{*}$ & $0.16^{*}$ & $0.04^{*}$ & \\
\hline Medium economic level: age & & & $0.26^{*}$ & & 0.09 & $0.16^{*}$ & & & & $0.03^{*}$ \\
\hline High economic level: age & & & $0.51^{*}$ & & $0.36^{*}$ & $0.33^{*}$ & & & & $0.12^{*}$ \\
\hline Constant & $136.03^{*}$ & $141.21^{*}$ & 109.43 & $81.34^{*}$ & $86.26^{*}$ & $121.82^{*}$ & $121.87^{*}$ & $76.09^{*}$ & $69.37^{*}$ & $64.12^{*}$ \\
\hline
\end{tabular}


increases with the economic level. The difference in scores between gender increases in favour of males with age. Scores decrease while age decreases and this variation is more important for high economic level for several dimensions such as RP, RE, MH and PCS. Health scores vary among social determinants and is not stable, being different for the various social determinants of individual.

\section{Conclusion}

This is a large and national study with a representative sample. The general population provided a detailed description of the quality of life of the different groups described by their demographic characteristics, and allowed for a comparison between them. Similar to a large number of studies, the HRQOL was measured through the SF-36 questionnaire. We have avoided over analysing the data and presented the population norms of SF-36 and the association of SF-36 domains with demographic and socio-economic variables only.

Our study highlighted a variety of HRQOL results among the different demographic characteristics of the Tunisian population. Our findings were promising for research on inequalities in health in Tunisia, since they showed a clear association between primary social variables (age and sex) and health. These findings may also be valid for other low- and middle-income countries. Our study provided population norms of SF- 36 that could be used for comparisons.

Quality of life differs with demographic characteristics in several countries in addition to regional differences within the country (6). This heterogeneity has been attributed to several factors such as gender inequality, aging and social inequality between regions. Efforts need to be made to reduce this inequality in regard to social and geographic factors $(2,5-7)$. Such studies should be repeated many times with a fixed interval in order to provide the possibility to focus on the progression of the quality of life across time.

\section{Funding: None.}

Competing interests: None declared.

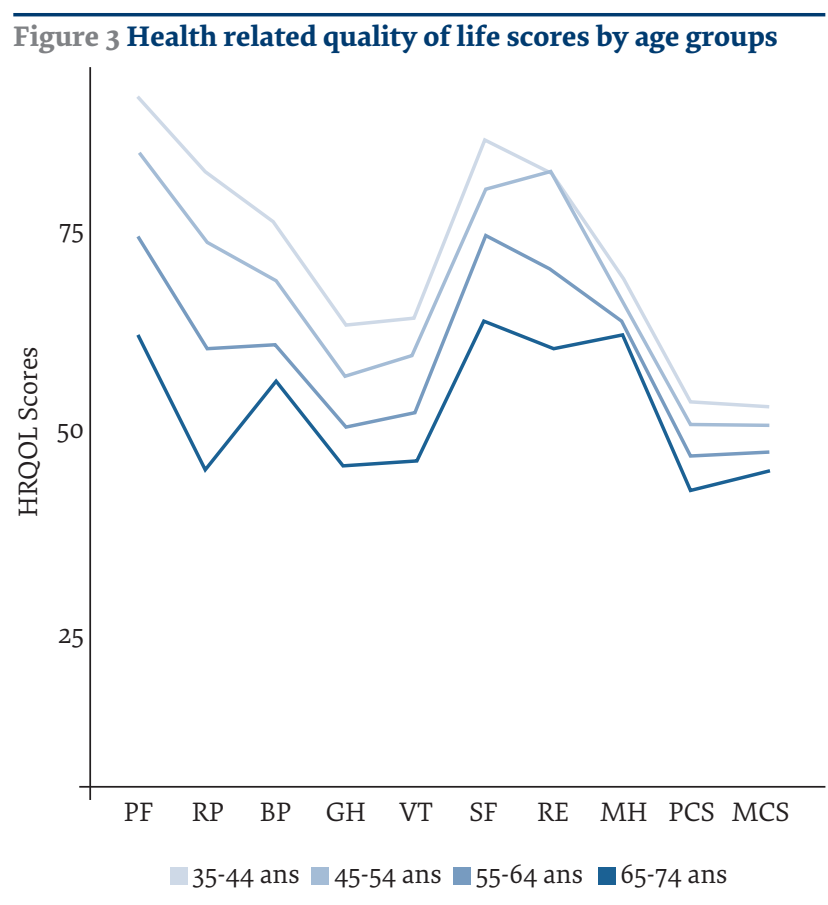

Figure 4 Health related quality of life scores by educational level

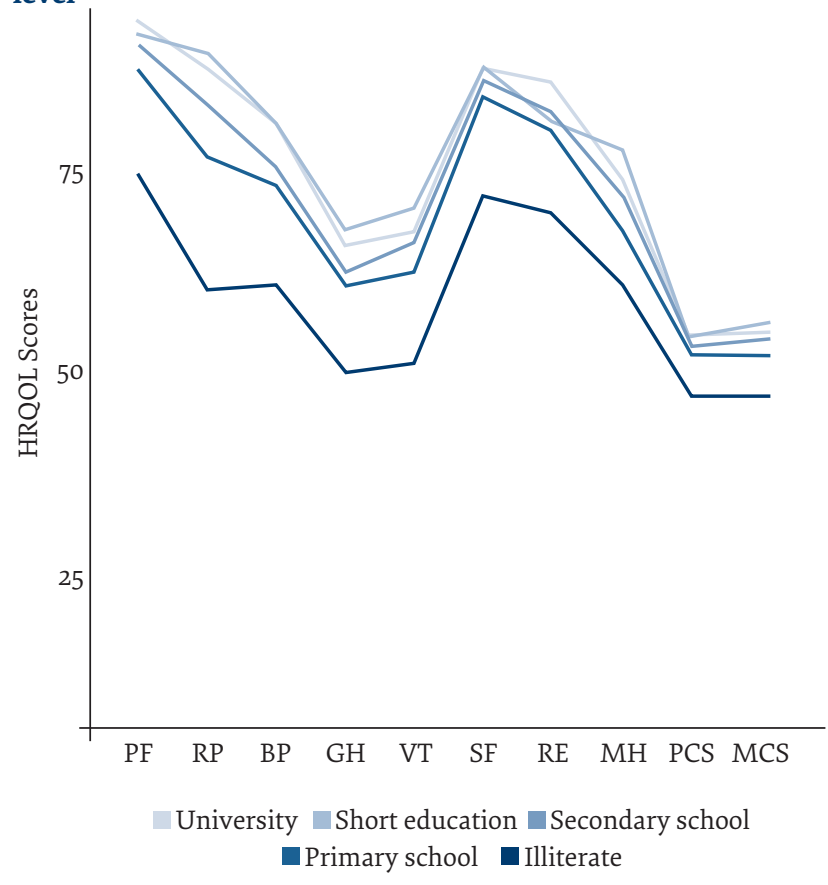

\section{Qualité de vie de la population tunisienne : analyse générale à l'aide du questionnaire SF-36 \\ Résumé}

Contexte : Le questionnaire SF-36 est l'instrument utilisé pour mesurer la qualité de vie des patients dans de nombreuses études cliniques nationales afin de décrire l'état de santé des populations et d'obtenir des données comparables dans ce domaine au niveau international.

Objectifs : La présente étude vise à obtenir des normes de population au moyen de la version tunisienne du questionnaire SF-36 et à évaluer le lien entre les scores correspondant à la qualité de vie liée à la santé et les caractéristiques démographiques de la population tunisienne.

Méthodes : Des entretiens en présentiel ont été menés dans le cadre d'une étude transversale en 2005 afin de recueillir des variables socio-démographiques et environnementales ainsi que des données auto-déclarées sur la qualité de vie. Un échantillon représentatif de 6543 participants âgés de 35 à 70 ans a été sélectionné. 
Résultats : Tous les scores présentent un niveau de cohérence interne et un coefficient de fiabilité élevés. Les niveaux des scores de la qualité de vie liée à la santé étaient associés aux caractéristiques socio-démographiques. Nous avons observé que ceux-ci diminuaient lorsque l'âge augmentait. Les moyennes pour les résumés des composantes physique et mentale étaient de $53+/-8$ pour les hommes et de 47,7+/-10 pour les femmes respectivement.

Conclusion : La présente étude est la première à s'intéresser à la population tunisienne générale et à mettre en lumière les facteurs associés à la qualité de vie liée à la santé dans le contexte tunisien.

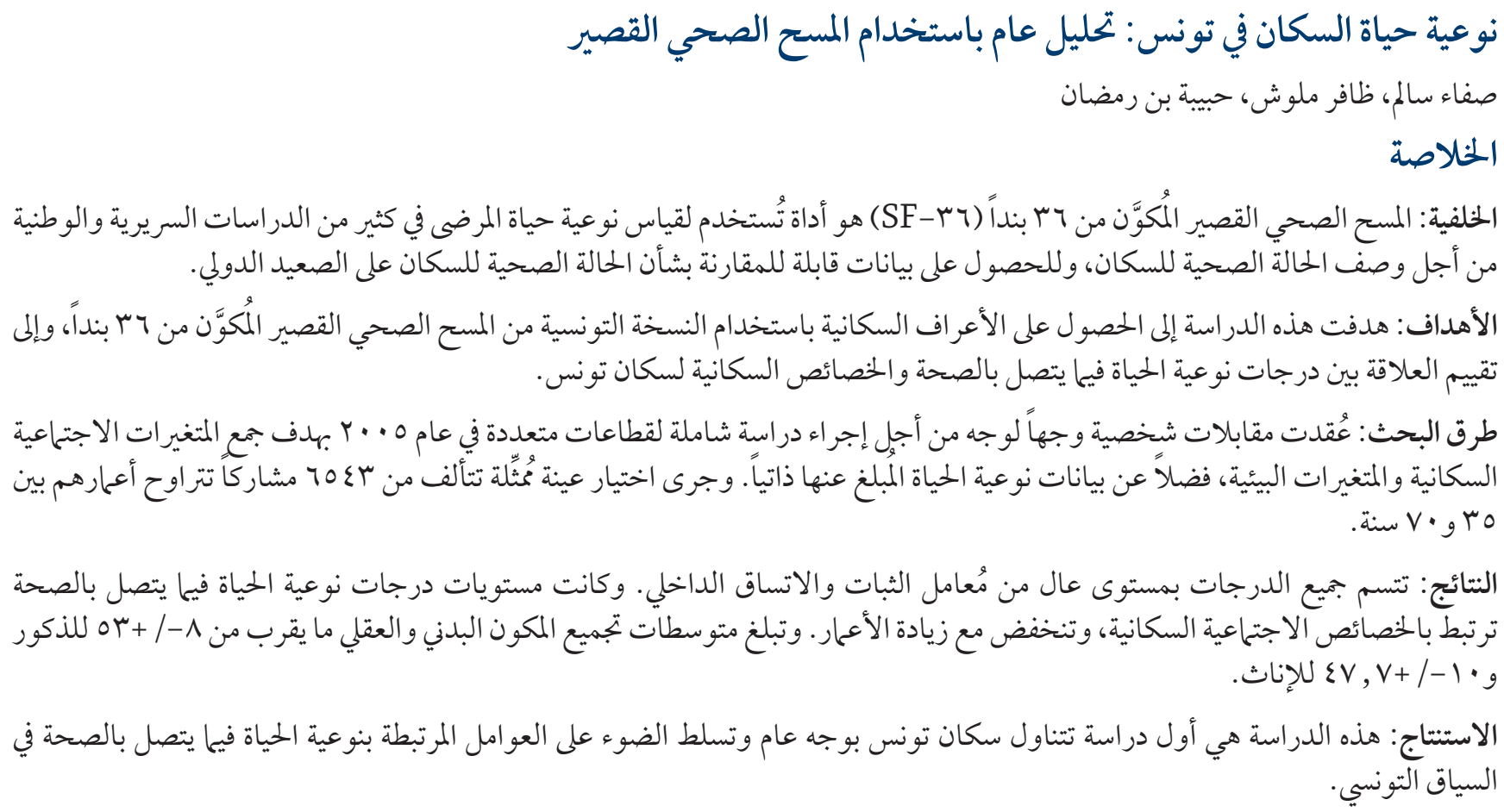

\section{References}

1. World Health Organization. Whoqol: measuring quality of life. Geneva: World Health Organization; 1997.

2. Jenkinson C, Stewart-Brown S, Petersen S, Paice C. Assessment of the sf-36 version_2 in the United Kingdom. J Epidemiol Community Health. 1999;53(1):46-50. http://dx.doi.org/10.1136/jech.53.1.46

3. Lee MD. Review of the short-form-36 (sf-36) health survey (2005).

4. Demiral Y, Ergor G, Unal B, Semin S, Akvardar Y, Kivırcık B, et al. Normative data and discriminative properties of short form 36 (SF-36) in Turkish urban population. BMC public Heath. 2006;6(1):247. PMID: 17029646 http://dx.doi.org/10.1186/1471-2458-6-247

5. Regidor E, Barrio G, de la Fuente L, Domingo A, Rodriguez C, Alonso J. Association between educational level and health related quality of life in Spanish adults. J Epidemiol Community Health. 1999;53(2):75-82. http://dx.doi.org/10.1136/jech.53.2.75

6. Sabbah I, Drouby N, Sabbah S, Retel-Rude N, Mercier M. Quality of life in rural and urban populations in Lebanon using SF-36 health survey. Health Qual Life Outcomes. 2003;1(1):30. http://dx.doi.org/10.1186/1477-7525-1-30

7. Richard JL. Validation et normes du SF-36 dans la population du Canton de Vaud. Institut universitaire de médecine sociale et préventive (2000).

8. Hsu HC. Gender differences in health-related quality of life among the elderly in Taiwan. Asian J Heal Inf Sci. 2007:1.

9. Tlili L, Lebib S, Moalla I, Ghorbel S, BenSalah F, Dziri C, et al. Impact de la pratique sportive sur lautonomie et la qualité de vie du paraplégique. In: Annales de réadaptation et de médecine physique, vol. 51, pp. Elsevier (2008).

10. Ghroubi S, Chaari M, Elleuch H, Guermazi M, Baklouti S, Elleuch M. Le devenir fonctionnel et la qualité de vie des ruptures de la coiffe des rotateurs non opérées. In: Annales de réadaptation et de médecine physique, vol. 51, pp. Elsevier (2008)

11. Zaghdoudi L, Homri W, Belaid S, Ben BM, Labbane R. (quality of life of patient with schizophrenia treated by conventional and atypical neuroleptics). Tunis Med. 2009;87(9).

12. UNDP. Human Development Report. Sustaining human progress: reducing vulnerabilities and building resilience. New York: UNDP; 2014.

13. Ware JE, Kosinski M, Keller S. SF-36 physical and mental health summary scales: a user's manual. Health Assessment Lab; 1994.

14. Taft C, Karlsson J, Sullivan M. Do sf-36 summary component scores accurately summarize subscale scores? Qual Life Res. 2001;10(5):395-404. http://dx.doi.org/10.1023/A:1012552211996 
15. Calcagno, Vincent. Package 'glmulti': Model selection and multimodel inference made easy. R package version 1.7 (2013).

16. Unit BE. South australian population norms for the short form 36 (sf-36) health status questionnaire. Adelaide: South Australian Health Commission; 1995.

17. Ware Jr JE, Sherbourne CD. The MOS 36-item short-form health survey (SF-36): I. conceptual framework and item selection. Med Care. 1992:30(6):473-483. PMID 1593914

18. Loge JH, Kaasa S. Short form 36 (SF-36) health survey: normative data from the general Norwegian population. Scand J Public Health. 1998;26(4):250-258. PMID 9868748 